\title{
SYMPOSIUM
}

\section{Management issues in CLD of prematurity}

\section{S Kotecha}

Arch Dis Child Fetal Neonatal Ed 2002;87:F2

CLD is one of the major problems in neonatal medicine. Clinicians struggle to provide optimal care because of limited evidence. Many questions still need to be answered; meanwhile many aspects of management will remain best practice rather than evidence based.

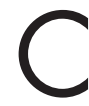
hronic lung disease of prematurity (CLD) remains a challenge for all in neonatal practice. Our ability to define it remains rudimentary despite many attempts to do so. ${ }^{1-4} \mathrm{In}$ practice, clinicians struggle to provide optimal care, partly because of limited evidence from randomised controlled trials especially for infants with established CLD. For the latter group, many questions remain unanswered: do home oxygen programmes improve outcomes including growth? Is oxygen saturation of $>94 \%$ better than a saturation of $<90 \%$ ? Are inhaled corticosteroids beneficial? What role do bronchodilators have? Do diuretics affect long term outcomes in the infant without involvement of the cardiovascular system? Many of these questions need to be addressed in the near future but until then we have to take a pragmatic approach to the management of infants at risk of developing CLD or with established CLD. Clearly it would be impossible to address all the issues associated with CLD, but this series of articles will concentrate on some areas that may be of particular interest to clinicians caring for infants with CLD.

Professor Greenough tackles the use of mechanical ventilation in preterm infants to decrease barotrauma or volutrauma. There have been many advances in ventilator types and modes and these are discussed in detail, with evidence given for and against each strategy. Many of the studies currently are far too limited to categorically recommend one mode over another. Even where several randomised controlled trials have been completedfor example, with high frequency oscillatory ventilation-concerns remain with outcomes such as air leaks and intracranial haemorrhages.

Could better monitoring result in optimal use of mechanical ventilators? Drs Schibler and Frey introduce us to the use of pulmonary lung function tests in ventilated preterm infants. These have largely been confined to research projects, and commercially available monitors have limitations not least because of the air leaks associated with uncuffed endotracheal tubes used in newborn infants and with the mechanical characteristics of the ventilatory circuits. Of particular interest to clinicians are lung volumes-for example, associated with hypoplasia. Newer methods such as the use of SF6 may have a role to play in assessing not only lung volumes but also distribution of gas in various parts of the lungs. Such methods may be useful for determining the optimal ventilatory settings, but, as the authors point out, the limitations of these methods need to be considered when interpreting the results.

In infants with established CLD, home oxygen programmes and prevention of pulmonary hypertension are the main focus of the next two articles. Home oxygen programmes appear to have improved the outlook of infants with CLD with particular regard to growth and prevention of pulmonary hypertension. Studies currently under way-for example, the BOOST in Australia-may provide further information on whether infants may benefit from lower oxygen saturations as opposed to the currently recommended saturations, which range from $\geqslant 92 \%$ to $\geqslant 94 \%$ after the first few weeks of life when the risk of retinopathy of prematurity is the greatest. It is essential that any such comparisons between low and high saturations also assess long term neurological outcomes to ensure that relatively hypoxic conditions do not adversely affect the child's long term development. Lessons clearly need to be learned from the trials on the use of corticosteroids in preterm infants. Oxygen remains a potent vasodilator of the pulmonary arteries and appears to have had an impact on pulmonary hypertension. Professor Abman discusses the pathophysiology of this condition and its management. In severe forms of this disease, which is now only rarely seen, other associated conditions should be sought and treated, and, if all else fails, more invasive tests may be indicated.

In this series of articles, we have covered areas that may be of particular interest to the reader. This is not to say that other areas are of less importance-for example, use of drugs and nutrition. Many infants are prescribed the former, often empirically, and the latter is likely to benefit both somatic and lung growth. The articles have attempted, and we hope succeeded in, giving the reader pertinent information on four important areas of CLD. Many questions remain to be answered, but until then many of the aspects of the management of infants with CLD will remain best practice rather than evidence based.

\section{REFERENCES}

1 Northway W, Jr, Rosan R, Porter D. Pulmonary disease following respirator therapy of hyaline-membrane disease: bronchopulmonary dysplasia. N Engl J Med 1967:276:357-68

2 Bancalari E, Abdenour GE, Feller R, et al. Bronchopulmonary dysplasia: clinical presentation. J Pediatr 1979:95:819-23.

3 Jobe AH, Bancalari E. NICHD, NHLBI, ORD workshop summary: bronchopulmonary dysplasia. Am J Respir Crit Care Med 2001; 163:1723-9.

4 Kotecha S, Silverman M. Chronic respiratory complications of neonatal disorders. In: Landau LI Taussig LM, eds. Textbook of pediatric respiratory medicine. Chicago: Mosby, 1999:488-521. 